\title{
THE MEASURING CHANNELS CALIBRATION IN THE INDUSTRY
}

\author{
Oleg Sulyma, PhD Student; Mykola Mykyichuk, Dr. Sc., Prof., \\ IrynaZinchuk, Lecturer, e-mail: mykolamm@ukr.net \\ Lviv Polytechnic National University, Ukraine
}

\begin{abstract}
The trend of development of measuring equipment in particular measuring systems and technological processes leads to the need of measuring the temperature without dismantling the primary transducers and stopping the technological process in a very wide range and with high accuracy. The choice of methods and means of measurement depends on such factors as the value of measured temperature, necessary accuracy of measurement, conditions of measurements, and operation of the investigated object.

The topicality of the problem is that industrial metrology operates the temperature measuring systems, transducers of which are deployed in such a way that their dismantling is impossible due to the specifics of the process or dangerous for operators' life (nuclear power plants). Such measuring systems, due to the specifics of their application, are not metrologically provided in the periods between major repairs of controlled objects, thus violating the unity of measurements.
\end{abstract}

Key words: Measuring channel; Metrological support; Measuring system; Thermocouple; Drift of characteristics.

\section{Introduction}

Measuring channels, which are parts of the system, namely the function from obtaining the measured value to ensuring the measurement result, represent the main component of measuring systems. Limited knowledge of the processes of destabilization of the properties of thermometric materials, and increasing instrumental errors of thermoelectric thermocouples inhibit the further development of thermometry.

The study of the processes occurring in thermometric material and causing operational changes in the transformation function has drawn attention to classical thermodynamics, which has long been considered as the theoretical basis of thermoelectricity. Processes in thermometric matter determine the function of thermocouple conversion. In terms of temperature measurements, as well as the action of a significant number of factors affecting the thermometric material, the latter is constantly changing.

The approach for estimating the result of thermocouples measurements can be based on both the theory of errors, which is quite well developed in metrology and the theory of uncertainty, which has recently spread in Europe. In general, the physical sources of measurement uncertainty coincide with the sources of measurement inaccuracies, which are also sources of errors in the theory of errors $[1,2]$.

The choice of method and means of measurement depends on such factors as the value of the measured temperature, necessary accuracy of measurement, conditions of measurements, and operation of the investigated object.

\section{Drawbacks}

The analysis of issues of improving the temperature measurement accuracy at continuously-operated objects without dismantling the primary transducers and interrupting the technological process is not resolved yet. In particular, the permanent temperature monitoring with means of the transducers installed in the measuring channels has to be provided in place.

\section{Goal}

Solving the problem of nonstop measuring temperature by the primary transducers without dismantling measuring channels of the complex technological facilities.

\section{Exact Methods of Measuring and} Monitoring the Temperature

The first method deals with the measurement of resistance of sensitive elements by replacing their resistances with a multivalued reference measure of resistance, eliminates the mentioned shortcomings, optimizes the measurement process, and measures the resistance and TEMP are provided/not provided by the metrological supervision with the necessary accuracy [3].

The second method involves the temperature measuring channels and consists of the implementation of the reference voltage calibrator for thermoelectric sensors $[4,5]$.

It should be noted that the first method defines the actual resistance of sensitive elements and simultaneously specifies temperature in the working volume by monitoring the actual resistance of the reference thermometer. The research electric scheme is shown in Fig. 1. In this case, the reference $R_{t N}$ and two operating resistance thermometers $R_{t x 1}$ and $R_{t x 2}$ are deployed in a chamber with a temperature controller, which in turn sets 5-6 temperature values within the measurement range. The measurements are carried out in a closed cycle, i.e. at each set of temperatures, the voltage comparator is balanced first on $R_{t N}$, and then with the help of multivalued measure of electrical resistance " 1 " of accuracy class $0.02 U_{M O}$ readouts (voltage drop on the multivalued measure of electrical resistance is balanced and returned to $R_{t N}$, thereby controlling the current's stability in the thermometer's circuit. The actual value of resistance of the reference thermometer sensitive element is determined from the measurements by (1), in which, unlike the classical potentiometric method, the value of the reference measure is not multiple of 10 but is equal to the resistance value: 


$$
R_{t N_{1}}=R_{M R} \frac{U_{t N}}{U_{M R}},
$$

where RMR is the value of resistance on the decade switches' multivalued measure of electrical resistance "1", taken from balancing UMR:

$$
\delta_{R_{t N}}= \pm\left(\delta_{R_{M i}}+\delta_{S M}+\delta_{0}\right)
$$

here $\delta_{R_{M i}}$ is equal to $0.02 \%$ for measures of resistance type P4831; $\delta_{S M}= \pm 2 K\left|\frac{U_{t_{N 1}}-U_{M R}}{U_{M R}}\right|$ is the substitution method error; $K=0.0005$ is an accuracy class of the voltage comparator; $\delta_{0}= \pm \frac{0,5 \Delta U_{V C}}{U_{M R}} \cdot 100 \%$ is an error of reference voltage comparator; $\triangle U V C$ is the price of the degree of the lover decade of the voltage comparator.

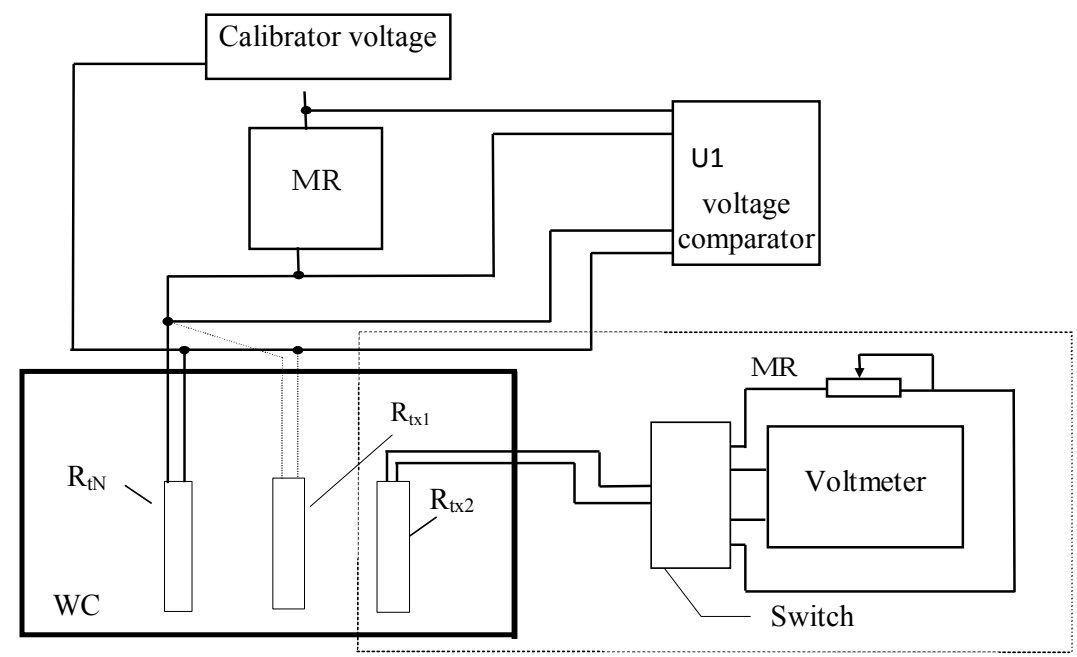

Fig. 1. Block diagram for determining the actual value of the resistance of the studied sensitive elements

While estimating the error components $\delta_{R_{t N}}$, we have set that $R_{t N}=R_{M 1}(1 \pm 0.0002) \Omega$. Then, based on the $R_{t N i}$ values obtained from (1), the actual value of the operating temperature at the controlled point is defined with help of Table [6]. Then the resistance store $R_{t x 1}$ is connected to the voltage comparator (shown in dotted line in Fig. 1), after which the output voltage $U_{t N}$ and $U_{t x 1}$ are measured in a closed cycle; the obtained readouts are applied to determine the controlled temperature.

$$
R_{t x_{1}}=R_{t N_{1}} \frac{U_{t x_{1}}}{U_{t N_{1}}},
$$

where $R_{t N 1}$ is the resistance of the reference thermometer calculated by (1). Based on $R_{t x i}$ computed by (3) with values from Table [6], the value of temperature $t_{x i}$ in the considered chamber, controlled by the thermometer $R_{t x 1}$. is determined. The absolute error of the operating thermometer at controlled temperature points is:

$$
\Delta t_{x_{i}}=t_{x_{i}}-t_{N_{i}} .
$$

After determining the temperature in the chamber with a reference thermometer $R_{t N}$, it needs to connect operating thermometer $R_{t x 2}$ to the reference ohmmeter and measure its resistance. Then disconnect $R_{t x 2}$ from the reference ohmmeter using the switch " $R_{t x 2}$ " and connect the measure of resistance that is the multivalued measure of electrical resistance " 2 " to it, set the same readouts of the reference ohmmeter altering its decades' values. The resistance values of the multivalued measure of electrical resistance " 2 " have to be equal to the resistance values of the working thermometer's sensitive element.

Then it is necessary to determine the limits (lower, upper) of the absolute calibration error of the sensitive element $\Delta_{C H E(H)}$ and determine the total error of each thermometer, consisting of two components caused by the sensitive element and the electronics. The average resistance determined for each temperature is set using the measure of resistance that's the multivalued measure of electrical resistance "2" and measure the analog or coded signal from the reference ohmmeter or PC for NART protocol.

The output signal is recorded and the measurements are repeated twenty times. The received temperatures are determined as well as the limits of the allowable error of the output signal of the electronic part $\triangle E P(L) T$ of the studied transducers. The total value of the main absolute error of the investigated thermocouple at controlled points is calculated:

$$
\Delta t_{x i}= \pm\left(\left|\Delta_{S E(L) T}\right|+\left|\Delta_{E P .(L) T}\right|\right) .
$$

Temperature calibration of the measuring channel that consists of calibration of the primary sensitive element (thermocouple) without dismantling it from the object of control. The structural measurement scheme is shown in Fig. 2. 


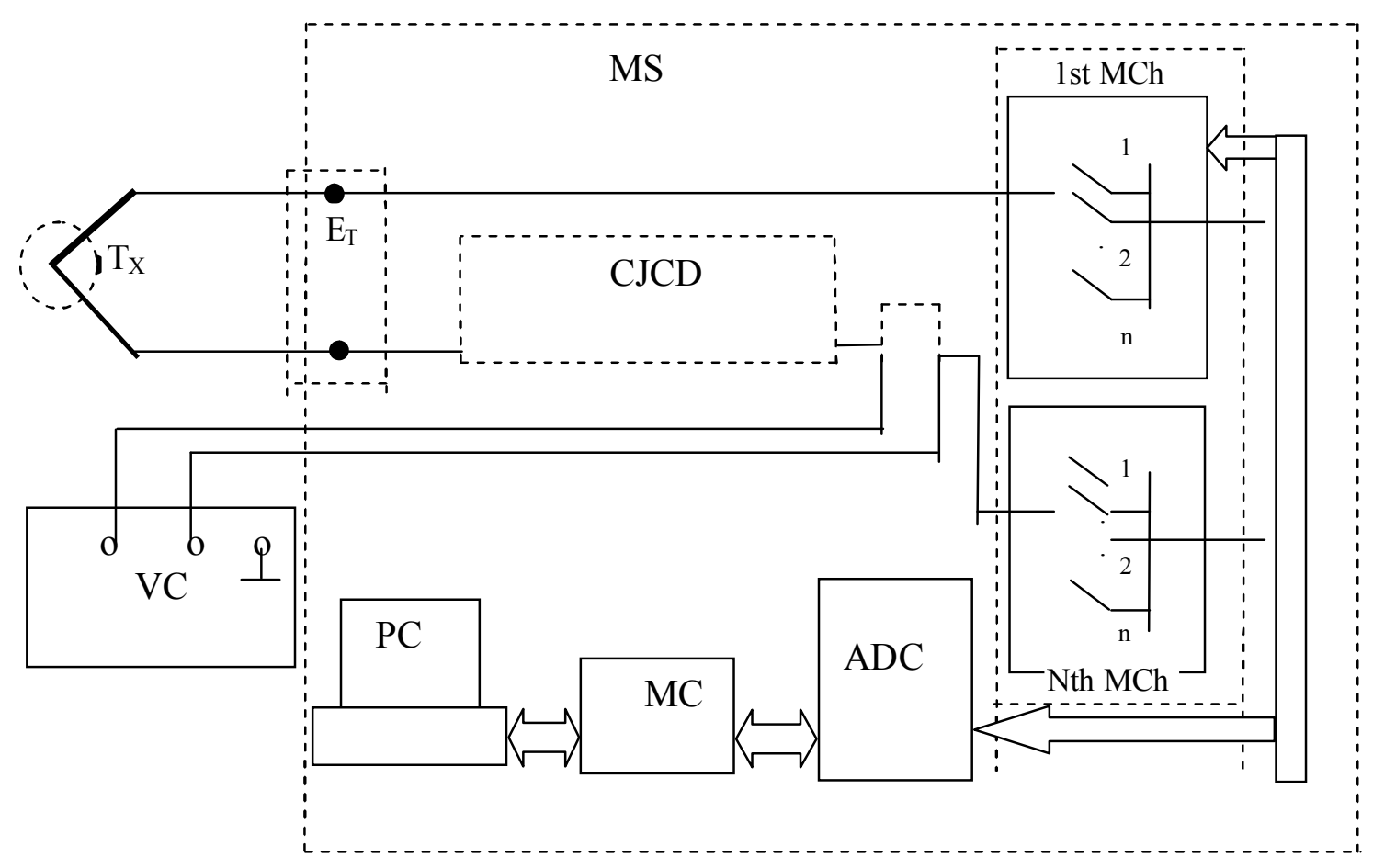

Fig. 2. The structural measurement scheme for controlling the temperature in the measuring channels with thermoelectric sensors: AS is an analog switch; MC is a microcontroller; CJCD is a cold junction temperature-compensation device, VC is a voltage calibrator

At the 1st stage of measurement, the so-called zero calibration is carried out. At the 2 nd stage of measurement, the actual temperature set at the facility is determined. For measuring channel temperature with a thermoelectric sensor according to the scheme of experimental studies, the voltage $U$ supplied to the input of the analog switch, and thus to the analog-to-digital transducer input will be determined according to:

$$
U=e_{T}+e_{Z T}+U_{O V}+U_{V C},
$$

where $e_{T}$ is the TEMP of thermocouple, which depend on the actual temperature $T$ of the controlled object, i.e. the temperature $T_{x} ; e_{z t}$ is parasitic TEMP caused from dissimilarity of extending and thermocouple wires; $U_{O V}$ is output voltage imbalance of the bridge compensation circuit; $U_{V C}$ is the output voltage of the voltage calibrator monitoring the measuring channel with help of the thermoelectric transducer. Hence, the absolute error $\Delta t_{M C}$ of the measuring channel is defined as:

$$
\Delta t_{M C}= \pm\left(\Delta_{T P}+\Delta_{Z T}+\Delta_{A D C}+\Delta_{C}+\Delta_{V C}\right)
$$

where $\Delta_{T P}$ is a thermocouple error; $\Delta_{Z T}=e_{Z T}$ is an error due to the dissimilarity of extending and thermocouple wires; $\Delta_{A D C}$ is an ADC quantitation error; $\Delta_{C}$ is the computing error.

It should be noted that errors $\Delta_{Z T}$ end $\Delta_{\mathrm{SKHS}}$ are proportional to the error $\Delta_{T P}$, and in the sum of $\Delta_{T P}$ reach values substantially larger than the error of the sum of $\Delta_{C}$ and $\Delta_{A D C}$, which is qualified as the error of the electrical circuit $\Delta_{E T}$ of the measuring channel. The thermocouple error $\Delta_{T P}$ could be reduced by structural method, thus the main task seems to determine it, as it is above considered.

To reduce the error from dissimilarity of thermocouple and extending wires, the last was chosen appropriate to the particular type of the thermocouple. In such a way it can be neglected by the error of the cold junction temperature-compensating device. Previous consideration has admitted that the errors $\Delta_{Z T}$ and $\Delta_{S K H S}$ are minimized and the error $\Delta_{K}$ is neglected since it is at least thrice less compared with $\Delta_{T P}$ or $\Delta_{E T}$ as the voltage calibrator is a reference one.

Before determination of errors $\Delta_{M C}$ and $\Delta_{T P}$, it is necessary to connect the voltage calibrator to an analog switch, set the voltage calibrator to $0.000 \mathrm{mV}$, which corresponds to $0{ }^{\circ} \mathrm{C}$ and fix the obtained result of measurement.

Such measurements must be made when approaching this point from below and above 10 times. The calculation of deviations of the obtained value from $0{ }^{\circ} \mathrm{C}$ is an absolute error $\triangle \mathrm{ET}$. Then the voltage calibrator is connected (see Fig. 1) and at zero voltage values, the current readout of temperature is obtained. Next, with the help of a voltage calibrator, this value should be set, by changing the polarity of the voltage calibrator.

The value of $0{ }^{\circ} \mathrm{C}$ must be recorded. In practice, this value differs slightly from $0{ }^{\circ} \mathrm{C}$. From the latter, it is 
necessary to subtract $\Delta_{E T}$, which is the absolute error of the studied thermocouple (transducer) $\Delta_{T P}$.

Note: The required measurements are relevant only at a stable temperature of the monitored object.

\section{Conclusions}

The considered methods for monitoring the temperature of the measuring channels of measuring systems with the deployed thermoelectric and thermo resistive sensors to which metrological supervision extends and which does not ensure with necessary accuracy in operation conditions allow without dismantling the mentioned channels:

- to increase the accuracy of temperature measurement by $17 \%$;

- to optimize the measurement process;

- eo carry out metrological verification of measuring channels and the specified transducers deployed in them.

\section{Conflict of Interests}

The authors state that there are no financial or other conflicts regarding the work.

\section{References}

[1] K. Sakharov, Yu. Parfenov, L. Zdukhov, B. Titov, V. Turkin, "Problems of the metrological support of measurements of the parameters of optical signals and devices in radar analog nanophotonic systems", Measurement Techniques, vol. 55, no. 2, 2012. doi:10.1007/s11018-010-9579-6

[2] A. Glazov, A. Ivanov, V. Kravtsov, A. Pniov, "Metrological support of measurements of the parameters of fiber-optic data transmission systems", Measurement Techniques, Vol.53, Iss.7, pp.789-797, 2010. doi:10.1007/ s11018-010-9578-7

[3] A. Buryachenko. Metrological support of measurements fot measuring channels calibrations with airxcraft engine regulator, Aerospace technic and technology, iss.7,p p.151-156, 2019. doi.org/10.32620/aktt.2019.7.22

[4] V. Dmytriv, M. Mykyychuk, I. Dmytriv, T. Dmytriv, "Analytical model of the measurer of thermoanemometric type of kinematic parameters of the biphasic pulsing flow", Measuring Equipment and Metrology, vol.80, iss.3, pp.46-52, 2019. doi.org/10.23939/istemtm2019.03.046

[5] V. Dmytriv, I. Dmytriv, T. Dmytriv, "Research in the thermo-anemometric measuring device of pulse flow of the two-phase medium", in Proc. 17th International Scientific Conference: ENGINEERING FOR RURAL DEVELOPMENT. University of Life Sciences and Technologies Faculty of Engineering, Proceedings, no. 17, Jelgava, Latvia, 2018, pp.898-904. doi:10.22616/erdev2018.17.n200

[6] EN 60584-1:2013 Interstate standard. Thermoelectric transducers. Nominal static characteristics of the transformation, 2013. 